\title{
XXIII. New analytic formula and table of an increasing life annuity; with remarks on the surrender of life assurance policies at proprietary offices
}

\author{
Mr. J.B. Benwell
}

To cite this article: Mr. J.B. Benwell (1821) XXIII. New analytic formula and table of an increasing life annuity; with remarks on the surrender of life assurance policies at proprietary offices , Philosophical Magazine, 57:275, 161-168, DOI: 10.1080/14786442108652477

To link to this article: http://dx.doi.org/10.1080/14786442108652477

冓 Published online: 27 Jul 2009.

Submit your article to this journal

山 Article views: 3

Q View related articles ¿ 


\title{
[ 161 ]
}

XXIII. New analytic Formula and Talle of an increasing Life Annuity; with Remarks on the Surrender of Life Assurance Policies at Proprietary Offices. By Mr.J. B. Bewwric, Hoxton.

\author{
To Mr. Tilloch.
}

Sir, - $T_{\text {Hx accompanying table, computed from real observa- }}$ tions and the probabilities of life, is applicable to a description and species of life annuity transaction, by no means common, and scarcely, under any form whatever, practised in this country; and any thing new or novel of this kind $I$ an inclined to imagine will be interesting to, and invite the attention of societies engaged and concerned in this particular and important branch of human oeconomy. On a former occasion I recommended the subject to their notice; and I do it now with the same persuasion of its being, by an easy extension of application, as suitably adapted to the views and purposes of individuals, as any other mode of assurance, and of its becoming at some future period in more general recurrence. I do not expect any credit for the suggestion proposed, because we know that, in the commerce of life, according as interest and gain may impart a colouring to the medium an object is seen through, it is either abandoned, or pursued with avidity. Now it happens in the instance under review, that the influence of this ordinary spirit and idol of general adoration is to be perceived: for the Offices (I speak in reference to the companies constituted solely on the trading system) in rather indirect terms and manner convey the inference gathered, that it is, as we may suppose, not a profitable speculation to them, or, more figuratively, the sweets of the hive are not exactly found in this track; and since the legislature has anticipated their privileged means in this respect, what comparatively they can do, but poorly compensates for the incidents attendant; for it is to be remarked, that it is not among a few independent but general mass of lives combined, that the caleulations of contingencies are founded and applied; and as an apparent consequence, the latter condition may be expected to partake in a higher degree favourable in the determination of the happening and failing of events grenerally, as well as in reference to the claims that may in probability of expectation successively terminate.

These reflections naturally lead me then to inquire into the motive, why most of the chartered companies display and hold forth their legislative powers enrolled and enabling them to speculate in the grant and purchase of annuities, on just and equitable principles, when at the same time they evince a colduess,

Vol. 57. No. 275. March 1821 . X neglect 
neglect and indifference to encourage and promote any emendation or extension devised in seeking out the ramifications of theory subordinate to practice. Where then is the consistency, in what is contained the policy of the immediate supporters of these schemes, or the engagements formed therein with them? If indeed they are to be identified with a character for disinterestedness, liberality and ingenuousness, and assuredly connive not at the intervention of stratagem, let them cease longer to hold out beacons deceptive, as well as those false lights that dazzle in the presence, but do not inform and direct the mind of the beholder, if all this be through a cause inadvertently, and not of design to impose on or delude the unwary. In glancing over these observations, it is likely they will be accounted matter of assertion unsupported by testimony of fact: that this may not supervene, I shall recognise, though among several, one tircumstance only as of recent occurrence in exemplification, and to show that they have not sprung out of a feeling of invective or prejudice, and as in the sequel there will be cause still to condemn the selfish and mercenary spirit by which such of these public bodies are actuated.

In the course of reference made to me on the subject of contingent property, I was lately requested to report upon the value of three policies of assurance, effected in certainly a very popular and fourishing concern : I allude not to the Equitable, for there in all its affairs we find a conscientious and scrupulous regard paid to the equitable claims and interest of all its members indiscriminately alike : thus a true parent of nature marks no distinction in the being of a common family and stock.

We will now briefly state the terms and dates of these policies when effected; they are as under-the life now in forty-first year of age :

$$
\begin{array}{lllllllll}
1 . & \ldots & 500 & \ldots & 12 l . & 5 s . & 5 d . & \ldots & 1806 \\
2 . & \ldots & 500 & \ldots & 12 & 15 & 5 & . & 1808 \\
3 . & \cdots & 1000 & \cdots & 26 & 2 & 6 & \cdots & 1809
\end{array}
$$

In the proposed consideration here it is necessary merely to remark, that upon a direct appeal of the party at the office to ascertain the sums that would be returned in lieu of the claims cxisting on a surrender being made conformably with the usual practice-they were the following nominally:

$$
1 \ldots 30 l \quad 2 \ldots 23 l \text {. } 3 \ldots 40 l \text {. }
$$

However, it may be replied by those in the conduct and management of the offices, that the sum the directors may, in caprice, think proper and choose to offer when they cancel a policy, 


\section{of an increasing Life Annuity.}

is no question of specific relation to the mathematical value ; but let them understand, that every person so conversant with this subject is enabled to say, and knows as well as they, what compensation should at any time be returned; and what as parties to a contract, at least construed in equity, they are bound by ties of ordinary faith to give the assured member. Let us now contrast the preceding sums, and equal but to $93 l$. as what would be allowed in a propriety concern : let us, I say, compare the extraordinary difference in the value of an assurance equal in amount effected with the Equitable under almost precisely similar circumstances; viz. on the same life for $2000 l$. in 1809 , at an annual payment of $52 \mathrm{l} .4 \mathrm{~s}$. ; but it is right to add, there is on this a bonus of $600 l$. were it now a claim; as also of $150 l$. on policy No. l, as I omitted to state. For the policy existing at the Equitable, then, the trustees will refund the sum of $514 l .9 s$. if surrendered any time before the annual premium due should be paid : so, allowing for this, the sum nominally offered is $566 l .13 s$. It is of no utility here to urge any thing as to the variation of the premiums, they being in the one case upon a single assurance, in the other apportioned upon three separately ; it is the aggregate of the annual premiums and relative amount of assurance we are directed to, and that strikes as dissonant to reason; it is an instance, in which the mind discriminates truth and error from derived intuitive evidence, and not enveloped in the mists of sophistry.

Further concerning these two values: as referred to the first announced, the discordant medium or discrepancy between them is not easily, if at all, explicable by any order or process of computation on which such kind of deductions should ever be regulated and assumed as the only infallible in result. We may next, as not without its use, since elucidation is the mean intended, proceed to a different view of the subjeet; for, by placing the same proposition in new and opposite directions, we see clearer what is either apposite or inconsistent. It appears then on the three former-described policies, the gross amount of premiums paid is (663l. $11 s .8 d$., or equal to 15 , on the first; 13 , on the second; 12, on the last; and these, if calculated as distinct annuities at 5 per cent., the first payment of each being made when the policy was opened, and so on at the beginning of every year succeeding, in which the life, it is considered, will be a claim-the equivalent amount is $952 l .3 \mathrm{~s} .6 \mathrm{~d}$. that is, equal in value now to an annuity of $47 l .12 s .2 d$. certain, to continue for ever, or in perpetuity at the same rate of interest, and in consideration of having received which, it is that the office proffers in-signifi$\mathrm{X} 2$ 


\section{Analytic Formula and Table of an increasing Lije Annitity.}

cant return the sum of barely $93 \mathrm{l}$. Is this then a precedent distinguished by any principle of liberality? is it distinguished by a feature of justice? It would, I imagine, be an excess of candour to confess as much; but nay, more than this unjustly it seems, that at some offices there is a refusal to cancel at all, or rather to make any return whatever of the premiums received; and thus, were it not through the instrumentality of those trafficking in such things, it would be inpossible to recover a single shilling of the immense sums so expended, and which, under the peculiar circumstance, would inevitably be sacrificed without any collateral advantage acquired. An observation occurs now, and though implied, yet should he expressly so, to the credit of the Society animadverted upon, it does then at recurring intervals divide with its members a part of the enormous gains of profit made; they do this in reality, and as what is falsely insinuated in many, where the scale of premiums is actually the same as at the Equitable, and yet never add a mite beyoud the sum assured; and with this fact so glaring, it bewilders one to think how they should be able to effect any policies with the public at all, excepting by the medium of a corrupt influence, or a courtesy tantamount to servility which their most interested and strenuous supporters may command in the world. If in general this is the arbitrary and unfair practice dictated in all the subscription Societies, how are we to applaud or even give credence to the reiterated professions made with unblushing effrontery, that in all negotiations with the public, both its " interest and convenience are considered," being in all their proceedings actuated by a strict sense of rectitude and of honour ?

I have in the present paper intentionally abstained from mixing up any extraneous calculations with it: by resolving algebraic into numerical values, the enunciation is simply apparent and intelligible to the general reader. I must now, in conformity with my original design, revert thereto, and introduce the table itself and auxiliary means of construction stited accordingly. 


\section{[ 165$]$}

Table of the Value of an Annuity of 1l. increasing in Order of the Series 1.2.3.4. .. n. on a Life at every Age from Three Years to extreme Duration. Computed from Olservations of $M$. DE PARCreuX.

\begin{tabular}{|c|c|c|c|}
\hline Age. & 3 per Cent. & $3 \frac{1}{2}$ per Cent. & 4 per Cent. \\
\hline 3 & 458,393 & 389,494 & 333,422 \\
\hline 4 & 463,367 & 394,275 & 337,923 \\
\hline 5 & 464,747 & 396,027 & 339,846 \\
\hline 6 & 464,227 & 396,174 & 340,404 \\
\hline 7 & 462,201 & 395,042 & 339,870 \\
\hline 8 & 459,116 & 393,018 & 338,564 \\
\hline 9 & 455,451 & 390,488 & 336,841 \\
\hline 10 & 450,679 & 387,006 & 334,288 \\
\hline 11 & 444,794 & 382,562 & 330,899 \\
\hline 12 & 437,806 & 377,158 & 326,685 \\
\hline 13 & 430,743 & 371,679 & 322,397 \\
\hline 14 & 423,606 & 366,125 & 318,02 \\
\hline 15 & 416,399 & 360,497 & 313,585 \\
\hline 16 & 409,123 & 354,797 & 309,08 \\
\hline 17 & 402,261 & 349,445 & 304,853 \\
\hline 18 & 395,327 & 344,016 & 300,57 \\
\hline 19 & 388.319 & 338,511 & 296,244 \\
\hline 20 & 381,24 & 332,932 & 291,813 \\
\hline 21 & 374,555 & 327,683 & 287,657 \\
\hline 22 & 367,792 & 322,354 & 283,423 \\
\hline 23 & 360,951 & 316,945 & 279,108 \\
\hline 24 & 354,031 & 311,454 & 274,712 \\
\hline 25 & 347,036 & 305,881 & 270,23 \\
\hline 26 & 339,962 & 300,227 & 265,662 \\
\hline 27 & 332,813 & 294,49 & 261,016 \\
\hline 23 & $325,58 \mathrm{~g}$ & 288,672 & 256,286 \\
\hline 29 & 318,292 & 282,765 & 251,471 \\
\hline 30 & 310,922 & 276,782 & 246,571 \\
\hline 31 & 303,48 & 270,718 & 241,585 \\
\hline 32 & 295,969 & 264,575 & 236,513 \\
\hline 33 & 288,389 & 258,352 & $231,35.5$ \\
\hline 34 & 280,744 & 252,052 & 226,111 \\
\hline 35 & 273,035 & 245,674 & 220,782 \\
\hline 36 & 265,266 & 239,222 & 215,369 \\
\hline 37 & 257,439 & 232,695 & 200,872 \\
\hline 38 & 249,186 & 225,762 & 203,988 \\
\hline 39 & 242,07 & 218,775 & 198,035 \\
\hline 40 & 233,807 & 211,7403 & 192,018 \\
\hline 41 & 225,525 & 204,663 & 185,942 \\
\hline 42 & 217,235 & 197,551 & 179,813 \\
\hline 43 & 208,944 & 190,412 & 173,636 \\
\hline 44 & 200,662 & 183,254 & 167,42 \\
\hline 4.5 & 192,399 & 176,080 & 161,171 \\
\hline 46 & 184.501 & 168,019 & 154,900 \\
\hline
\end{tabular}




\section{[ 166 ]}

TABLE continued.

\begin{tabular}{|c|c|c|c|}
\hline Age. & 3 per Cent. & $3 \frac{\mathrm{I}}{2}$ per Cent. & 4 per Cent. \\
\hline 47 & 176,71 & $16 \overline{2,03}$ & 148,81 \\
\hline 48 & 168,863 & 155,147 & 142,75 \\
\hline 49 & 161,338 & 148.532 & 136,918 \\
\hline 50 & 154,173 & 141,93 & 131,076 \\
\hline 51 & 147,012 & 135,587 & 125,452 \\
\hline 52 & 140,164 & 29.493 & 120,037 \\
\hline 53 & 133,343 & 123,413 & 114,616 \\
\hline 54 & 126,573 & 117,355 & 109,195 \\
\hline 55 & 120,092 & I 11,542 & 103,981 \\
\hline 56 & 113,407 & 105,757 & 98,774 \\
\hline 57 & 107.037 & 100.013 & 93,583 \\
\hline 58 & 100,951 & 94,51 & 88,6 \\
\hline 59 & 94938 & 89,054 & 83,641 \\
\hline 60 & 89,01 & 83,655 & 78,716 \\
\hline 61 & 83,178 & 78,324 & 73,836 \\
\hline 62 & 77,455 & 73,075 & 69,013 \\
\hline 63 & 72,028 & 68,083 & 64,415 \\
\hline 64 & 66,723 & 63,187 & 59,891 \\
\hline 65 & 61,557 & 58,402 & 55,456 \\
\hline 66 & 56,697 & 53,888 & 51,26 \\
\hline 67 & 52,135 & 49,642 & 47,304 \\
\hline 68 & 47,87 & 45,663 & 43,588 \\
\hline $6 g$ & 43,9 & 41,951 & 40,113 \\
\hline 70 & 40.223 & 38,503 & 36,879 \\
\hline 71 & 36,709 & 35,205 & 33,775 \\
\hline 72 & 33,497 & 32,18 & 30,924 \\
\hline 73 & 30,462 & 29,316 & 28,218 \\
\hline 74 & 27,613 & 26,622 & 25,666 \\
\hline 75 & 24,96 & 24,105 & 23,277 \\
\hline 76 & 22,387 & 21,662 & 20,952 \\
\hline 77 & 20,033 & 19,421 & 18,814 \\
\hline 78 & 17,907 & 17,393 & 16,875 \\
\hline 79 & ] 5,902 & 15,475 & 15,039 \\
\hline 80 & 14,148 & 13,798 & 13,431 \\
\hline 81 & 12,537 & 12,258 & 11,95 \\
\hline 82 & 11,124 & 10,859 & 10,603 \\
\hline 83 & 9,686 & 9.47 & 9,263 \\
\hline 84 & 8,249 & 8,078 & 7,914 \\
\hline 85 & 6,953 & 6,821 & $6,69^{2}$ \\
\hline 86 & 5,807 & 5,705 & 5,607 \\
\hline 87 & 4,814 & 4,738 & 4,664 \\
\hline 88 & 3,79 & 3,735 & 3,684 \\
\hline 89 & 2,893 & 2,956 & 2,821 \\
\hline go & 2,126 & 2,103 & 2,08 \\
\hline 91 & 1,486 & 1,472 & 1,459 \\
\hline 92 &, 9567 &, 9499 & 943 \\
\hline 93 &, 4854 & ,4831 &, 481 \\
\hline 94 & zero. & zero. & zero. \\
\hline
\end{tabular}


Respecting these numbers and tabulated values, which $I$ have extended to other rates, but cannot well augment here, it is to be observed when at any time needed they are directed to be calculated by M. De Moivre's Hypothesis, from which some neat and appropriate formula has been investigated; but it is obvious the values deduced by it are only approximate to the true ones as found from the series of decrements of life; but this latter value however can be obtained, and, excluding the hypothesis, with greater ease, facility and accuracy of course, after the manner ensuing : first, having from real observations and for any order of increase determined the value of any such amnuity upon an advanced life, or beginning from au age near the end of the table, then having for any form chosen so determined the value at the age hegun with, we may thence assign in succession the value for every age intercepted, or that preceding, by aid of a subsidiary formula; and the operation being continued until the Table is completed, as also similarly for single, joint or longest of lives, the application may be generalized.

Eumerating therefore the formula of derivation. Of the order of numeral series in the table 1.2.3.4. \&c.

Let A signify the value of $1 l$. annuity on life $\mathrm{N}$, at age $n-1$. $B$, that at age $n$, but increasing by $1.2 ., 3 l$. Then the value of such annuity for life $\mathrm{N}$, is expressed by $\left(\mathrm{A}+\mathrm{B} \times \frac{a^{\prime}}{a z}\right)$.

If the annuity increases in the order 1.3.5.7; if $\mathrm{C}$ be its value at age $n$, then is whole value for $\mathrm{N}=2 \mathrm{~A}+(\mathrm{C}-1) \cdot \frac{a^{\prime}}{c x}=$ $\left(\mathrm{A}+\frac{2 \mathrm{~B} a^{\prime}}{a x}\right)$.

If increasing by the triangular series 1.3.6. 10 ; and $D$ be its value at age $n$, the value for $\mathrm{N}$ is $=\mathrm{A}+\frac{(\mathrm{B}+\mathrm{D}) a^{\prime}}{a x}$.

If it increases as the quadrangular series 1.4.9.16, the value for $\mathrm{N},=\mathrm{A}+\frac{(\mathrm{B}+2 \mathrm{D}) a^{\prime}}{a x}$.

Where in each theorem $\frac{a^{\prime}}{u x}$ is the expectancy of $1 l$, on life $\mathrm{N}$, a year hence. And, expanding by the combinatorial methods, other forms of series will be developed, and the formula of continuation applied. There are beside certain modifications fitting in practice, and some relative to the deduction of value in the case when an annuity is not for the whole term of duration, but temporary, or in remainder deferred; but these are not here admitting a full description.

This being the first table of the value of a life annuity increasing, 
creasing, faunded on any registered observations of mortality, that have as yet been published, the computist may lean with due confidence on its accuracy; it contains, I telieve, no error of moment, the numbers respectively were derived from a double operation pursued, logarithmically and by calculation, the former to check and verify the latter; and though this is indispensable, yet the process by logarithms is not invariably critically exact in any specific inquiry, as will happen in fact from such tables, having been mostly by interpolation of differences compiled and extended: when then any result occurs so, as dissonant, it is only to be settled by a distinct revision, the work collated anew, or by some independent mode of verification.

Of all the chartered companies established, they avowedly profess to blend with their customary the life annuity business in every species of variety and form : it is unaccountably strange, then, they evince not partially a wish to promote the cause of that science, by which it is, that the stability of their plans is to be determined, and even existence vitally depend; and if we look unto those mushroom societies spontaneously emerging out of obscurity, ignorance or corrupt motives, it is but too visibly beheld that the age of bubble scheme and system has revived again, or is rapidly reviving: it would be a truly laudable task for any one possessing abstractedly the means, to devote some portion of time and talent to the discussion of their principles, views and measures; if however, and I must so qualify the expression, they can sustain this test and scrutiny of examination. I am aware of what is to be done, but, much as inclined, the engagement is such that few cheerfully and with alacrity would undertake and prosecute it through all its minutiæ of condition.

Aske Terrace, Hoxton, Feb. 1821.

J. B. BENWELL.

XXIV. On the Solar and Lunar Periods. By Mr. JAmes UtTing, of Lynn Regis.

\section{To $M r$. Tilloch.}

Sir, - I have sent you for insertion in the Philosophical Magazine (if approved) a few extracts and remarks relative to the solar and lunar periods, as stated by modern astronomers, in order (in part) to meet the wishes of Mr.Yeates : as probably some of your astronomical correspondents are in possession of the means of a more extensive reference, my selections are but few, but I believe them to be the most correct published, agreeable to the present improved state of the science.

I remain, sir, yours truly,

Norfolk-street, Lynn Fegis, Feb. \&, 182].

James UTting.

M. DE 